\title{
Glucose-6-phosphate dehydrogenase deficiency and reduced haemoglobin levels in African children with severe malaria
}

Christian N. Nguetse ${ }^{1}$, Christian G. Meyer ${ }^{1,2}$, Ayola Akim Adegnika ${ }^{1,3}$, Tsiri Agbenyega ${ }^{4,5}$, Bernhards R. Ogutu ${ }^{6}$, Peter G. Kremsner ${ }^{1,3}$ and Thirumalaisamy P. Velavan ${ }^{1,2,7^{*}}$

\begin{abstract}
Background: Extensive studies investigating the role of host genetic factors during malaria associate glucose6-phosphate dehydrogenase deficiency with relative protection. G6PD deficiency had been reported to associate with anti-malarial drug induced with haemolytic anaemia.
\end{abstract}

Methods: A total of 301 Gabonese, Ghanaian, and Kenyan children aged 6-120 months with severe malaria recruited in a multicentre trial on artesunate were included in this sub-study. G6PD normal (type B), heterozygous (type $\mathrm{A}^{+}$) and deficient (type $A^{-}$) genotypes were determined by direct sequencing of the common African mutations G202A and A376G. Furthermore, multivariate analyses were executed to associate possible contributions of G6PD deficiency with baseline haemoglobin levels, parasitaemia and with severe malarial anaemia.

Results: Two hundred and seventy-eight children (132 females and 146 males) were successfully genotyped for G6PD variants. The overall prevalence of G6PD deficiency was $13 \%$ [36/278; $3 \%(4 / 132)$ female homozygous and $22 \%$ (32/146) male hemizygous], $14 \%$ (40/278) children were female heterozygous while $73 \%$ (202/278) were G6PD normal [67 \% (88/132) females and $78 \%$ (114/146) males] individuals. Multivariate regression revealed a significant association of moderately and severely deficient G6PD genotypes with haemoglobin levels according to the baseline data ( $p<0.0001$; G6PD heterozygous: $p<0.0001$; G6PD deficient: $p=0.009$ ), but not with severe malarial anaemia $(p=0.66)$. No association of G6PD genotypes with baseline parasitaemia.

Conclusions: In this study, moderately (type $\mathrm{A}^{+}$) and severely (type $\mathrm{A}^{-}$) G6PD deficiency showed significant association with lower haemoglobin concentrations at baseline in African children with severe malaria without leading to severe malarial anaemia. In addition, there was no association of G6PD variant types with parasite densities on admission.

Keywords: Glucose-6-phosphate dehydrogenase deficiency, African children, Severe malaria

\section{Background}

Malaria remains a major health problem, with approximately 3.2 billion people at risk. In 2015, WHO reported approximately 214 million cases and about 438,000 deaths occurring in the world with the highest morbidity and mortality rates observed in Africa, especially among

\footnotetext{
*Correspondence: velavan@medizin.uni-tuebingen.de

${ }^{7}$ Fondation Congolaise pour la Recherche Médicale, Brazzaville, Republic of Congo

Full list of author information is available at the end of the article
}

children under 5 years of age [1]. The control of the disease particularly in low transmission settings is the key target for malaria elimination [2] but such success is still a great challenge [3]. One approach to reduce the disease incidence is to block the transmission. Primaquine, a 8-aminoquinoline effective for both transmission blocking of Plasmodium falciparum and anti-relapse treatment against Plasmodium vivax has been recommended for many years by the World Health Organization (WHO) $[4,5]$. However, primaquine has a major drawback which limits its widely use. The drug is known to cause acute 
haemolytic anaemia in individuals with glucose-6-phosphate-dehydrogenase (G6PD) deficiency [6, 7].

G6PD is a key enzyme catalysing the first reaction in the pentose phosphate pathway and provides a reduced form of nicotinamide adenine dinucleotide phosphate (NADPH) to help cells to counterbalance oxidative stress [8]. G6PD deficiency is a common, X-linked hereditary enzyme deficiency affecting approximately 400 million people worldwide [9], mainly in malaria-endemic regions [10]. Among the 186 mutations identified until 2012 in the G6PD gene [11], the variants 376A (G6PD type B), 376G (G6PD deficiency type $\mathrm{A}^{+}$and 202A (severe G6PD deficiency type $\mathrm{A}^{-}$) are the most common ones. The deficiency types $\mathrm{A}^{+}$(moderately deficient) and $\mathrm{A}^{-}$(severely deficient) constitute up to $90 \%$ of reported G6PD variants $[8,12,13]$. Other mutations such as A542T, G680T or T968C have also been identified in parts of Africa and been suggested to contribute to G6PD deficiency. However, information available on these mutations is very scarce. For example, the T968C mutation has been reported to be common only in The Gambia [14] and Senegal [15]. Although, G6PD diagnostic enzyme tests are available, they are currently not widely used in most clinical studies, particularly because primaquine, is not regularly used in Africa. Any attempts to control malaria need to take into account also tertian malaria, which, although occurring rarely only in many parts of Africa, contributes to the world-wide malaria burden.

Previous case-control studies have reported an association of G6PD deficiency, in particular of the 202A variant, with an increased risk of severe malarial anaemia and a protection or reduced risk against cerebral malaria $[16,17]$. Therefore, it is interesting to assess the influence of this allele on the clinical presentation of severe malaria among the African children recruited into this study.

This study was a sub-study of a multicentre trial of artesunate conducted by the "Severe Malaria in African Children" (SMAC) consortium which assessed the non-inferiority of a simplified 3-dose regimen of intramuscular and intravenous artesunate [18]. The aim was to determine using direct sequencing the frequency distribution of G6PD deficiency in malaria children from Africa. Some exploratory analyses have also been performed to investigate the effect of G6PD genotypes on asexual parasitaemia, haemoglobin concentrations and severe malarial anaemia during admission.

\section{Methods}

\section{Study design and participants}

This study was a sub-study of the SMAC follow-up study. The SMAC study was an open-label, randomized, multicentre (Gabon, Malawi, Ghana, Kenya, and The Gambia), parallel-group, three-arm study to compare the anti-malarial activity and safety of three artesunate (ARS) dosing regimens in children with severe $P$. falciparum malaria. Patients were randomly assigned to one of three dosing regimens consisting of a total of $12 \mathrm{mg} /$ $\mathrm{kg}$ parenteral ARS: (i) $2.4 \mathrm{mg} / \mathrm{kg}$ intramuscular on admission and at 12, 24, 48 and $72 \mathrm{~h}$, (ii) $4 \mathrm{mg} / \mathrm{kg}$ intramuscular on admission and at 24 and $48 \mathrm{~h}$, and (iii) $4 \mathrm{mg} / \mathrm{kg}$ intravenous on admission and at 24 and $48 \mathrm{~h}$ post admission. Parasitaemia was assessed by thick blood smears at $6 \mathrm{~h}$ intervals and prior to the each dose of treatment for at least $48 \mathrm{~h}$ following the first dose of study drug. Malaria occurs holoendemically and transmission rates in all study countries are high and perennial.

The present study involved participants of the SMAC study from Gabon, Ghana and Kenya. Three hundred and one children aged 6-120 months with a diagnosis of $P$. falciparum infection (parasitaemia $\geq 5000$ parasites $/ \mu \mathrm{L}$ on initial blood smear) made using an alternative to conventional thick film examination (Lambaréné method) [19] were randomly selected from the SMAC follow-up study [18]. Blood samples $(400 \mu \mathrm{L})$ from all participants were collected in heparinized tubes. Specimens were stored at $-80^{\circ} \mathrm{C}$ for subsequent molecular analyses.

\section{Ethics statement}

The study was conducted in accordance with Good Clinical Practices, and approved by authorities for each study site (the Regional Ethics Committee in Lambaréné (CERIL) for Gabon, Committee on Human Research, Publication and Ethics, Kwame Nkrumah University of Science and Technology (KNUST), Kumasi, for Ghana and the National Ethics Research Committee, Kenya Medical Research Institute (KEMRI) for Kenya). Children were enrolled into this study if a parent or guardian was willing to provide written informed consent in accordance with local practice.

\section{G6PD genotyping}

Genomic DNA was isolated using QIAamp DNA mini blood kit (Qiagen, Hilden, Germany). A 968 bp fragment of the G6PD gene containing the polymorphisms 202G > A and 376A > G was amplified by PCR using primers $5^{\prime}$-GCCCCTGTGACCTCCCGCCA-3' (forward) and 5'-GCAACGGCAAGCCTTACATCTGG-3' (reverse). The main focus was directed only to these two variants although some other deficient genetic mutations such as A542T (Senegal $1 \%$, The Gambia $2.2 \%$ ), G680T (The Gambia $0 \%$, Senegal $0 \%$ ) and T968C (The Gambia $7.8 \%$, Senegal $10 \%$ ) have been reported at a substantially lower prevalences only $[14,15]$, and might have been present in this study population. However, they seem not to be responsible for the prevalence of G6PD deficiency in all parts of Africa [20]. Briefly, $10 \mathrm{ng}$ of genomic 
DNA were added to a $20 \mu \mathrm{L}$ reaction mixture containing $1 \times$ PCR buffer $(20 \mathrm{mM}$ Tris- $\mathrm{HCl} \mathrm{pH} \mathrm{8.4,50} \mathrm{mM} \mathrm{KCl,}$ $1.5 \mathrm{mM}$ of $\left.\mathrm{MgCl}_{2}\right), 0.125 \mathrm{mM}$ of dNTPs, $0.25 \mathrm{mM}$ of each primer and $1 \mathrm{U}$ Taq DNA polymerase (Qiagen, Hilden, Germany). The PCR was run on a PTC-200 Thermal cycler (M) Research, Waltham, USA). Thermal conditions after initial denaturation $\left(94^{\circ} \mathrm{C}, 5 \mathrm{~min}\right)$ were 35 cycles of $94{ }^{\circ} \mathrm{C}$ for $45 \mathrm{~s}, 65^{\circ} \mathrm{C}$ for $1 \mathrm{~min}$, and $72{ }^{\circ} \mathrm{C}$ for $1 \mathrm{~min}$. PCR reactions were completed with a final extension step of $72{ }^{\circ} \mathrm{C}$ for $5 \mathrm{~min}$. PCR products were visualized through electrophoresis on a $1.2 \%$ agarose gel stained with SYBR green I in $1 \mathrm{x}$ Tris-electrophoresis buffer $(90 \mathrm{mM}$ Trisacetate, $\mathrm{pH}$ 8.0, $90 \mathrm{mM}$ boric acid, $2.5 \mathrm{mM}$ EDTA).

Subsequently, PCR products were purified (Exo-SAPIT, USB, Affymetrix, USA) and directly used as templates for DNA sequencing using the BigDye terminator v. 1.1 cycle sequencing kit (Applied Biosystems, Foster City, USA) on an ABI 3130XL DNA sequencer. G6PD polymorphisms were identified by assembling the sequences with the reference sequence of G6PD (NG_009015.2) gene using the Codoncode Aligner 4.0 software (http:// www.codoncode.com) and visually reconfirmed from their electropherograms.

\section{Statistical analysis}

Data were analysed by using GraphPad Prism v. 5.0 for windows (GraphPad software, San Diego, CA). The effect of G6PD genotypes was determined on initial parasitaemia and haemoglobin values using a multivariate regression model. The children were classified in groups of normal, intermediate (female heterozygous) and deficient (hemizygous males and homozygous females) individuals. To evaluate the effect of G6PD genotypes on haemoglobin concentrations, the model included adjustment for age, gender, centre, weight, temperature and parasitaemia. To investigate the G6PD effect on parasitaemia, parasite densities were log-transformed and the model included adjustment for age, gender, centre, weight, haemoglobin levels and temperature. For the construction of the multivariate regression model, a subjective modelbuilding approach that excludes possible confounders such as gender, age and origin of study participants was applied. Kruskal-Wallis of One-way ANOVA with Dunn's Multiple Comparison and Mann-Whitney tests were used to determine the differences among categories. The level of significance was set to a $P$ value of 0.05 .

\section{Results \\ Patients}

According to the SMAC definition of severe malaria which perfectly reflects the policies of most African hospitals [21,22], the frequency of severe malaria syndromes at presentation were substantially different across the three study sites (Table 1). The majority of children fulfilled one or more criteria of the WHO definition of severe malaria [23, 24], which include severe anaemia (haematocrit of $<15 \%$ or $\mathrm{Hb}<5 \mathrm{~g} / \mathrm{dL}$ with a parasitaemia of $>10,000 / \mu \mathrm{L})$, hyperlactataemia $(\geq 5 \mathrm{mmol} / \mathrm{L})$, hyperparasitaemia ( $>250,000$ parasites $/ \mu \mathrm{L}$ ), hypoglycaemia (whole blood or plasma glucose $\leq 2.2 \mathrm{mmol} / \mathrm{L}$ ), and haemoglobinuria (urine that is dark red or black, with a dipstick that is positive for $\mathrm{Hb} /$ myoglobin). A description of children screened, recruited and genotyped for their G6PD status is shown in Fig. 1. From the 287 malaria children enrolled, the G6PD genotypes were available for only 278 children. One hundred and forty-six children (53\%) were males. The median age was 2 (IQR: $1-4$ ) years ranging from 6 months to 10 years with a mean haemoglobin value of $8.5( \pm 2.4) \mathrm{g} / \mathrm{dL}$.

\section{Prevalence of G6PD genotypes and associations with baseline variables}

Overall, 202 (73\%) children were classified as G6PD normal [type B; 114 (78 \%) males, 88 (67 \%) females], while $40(14 \%)$ were female heterozygous (type $\mathrm{A}^{+}$) and 36 (13\%) were G6PD deficient [type $\mathrm{A}^{-}$; 32 (22 \%) males hemizygous and 4 ( $3 \%$ ) female homozygous]. In Table 2, the genotype frequencies of G6PD for males and females

Table 1 Distribution of severe malaria syndromes by study centre

\begin{tabular}{lcccc}
\hline & All (\%) & Lambaréné, Gabon (\%) & Kumasi, Ghana (\%) & Kisumu, Kenya (\%) \\
\hline $\begin{array}{llll}\text { Severe malaria syndromes at admission } \\
\text { Respiratory distress }\end{array}$ & $10 / 278(4)$ & $1 / 108(1)$ & & $0 / 83(0)$ \\
Prostration & $55 / 278(20)$ & $4 / 108(4)$ & $43 / 87(10)$ & $8 / 83(10)$ \\
Cerebral malaria & $13 / 278(5)$ & $0 / 108(0)$ & $12 / 87(14)$ & $1 / 83(1)$ \\
General seizure & $20 / 278(7)$ & $4 / 108(4)$ & $13 / 87(15)$ & $3 / 83(4)$ \\
Severe anaemia & $26 / 278(9)$ & $5 / 108(5)$ & $20 / 87(23)$ & $1 / 83(1)$ \\
Jaundice & $21 / 278(8)$ & $0 / 108(0)$ & $16 / 87(18)$ & $5 / 83(6)$ \\
\hline
\end{tabular}

Children can appear in more than one category

${ }^{a}$ Missing data for some syndromes 


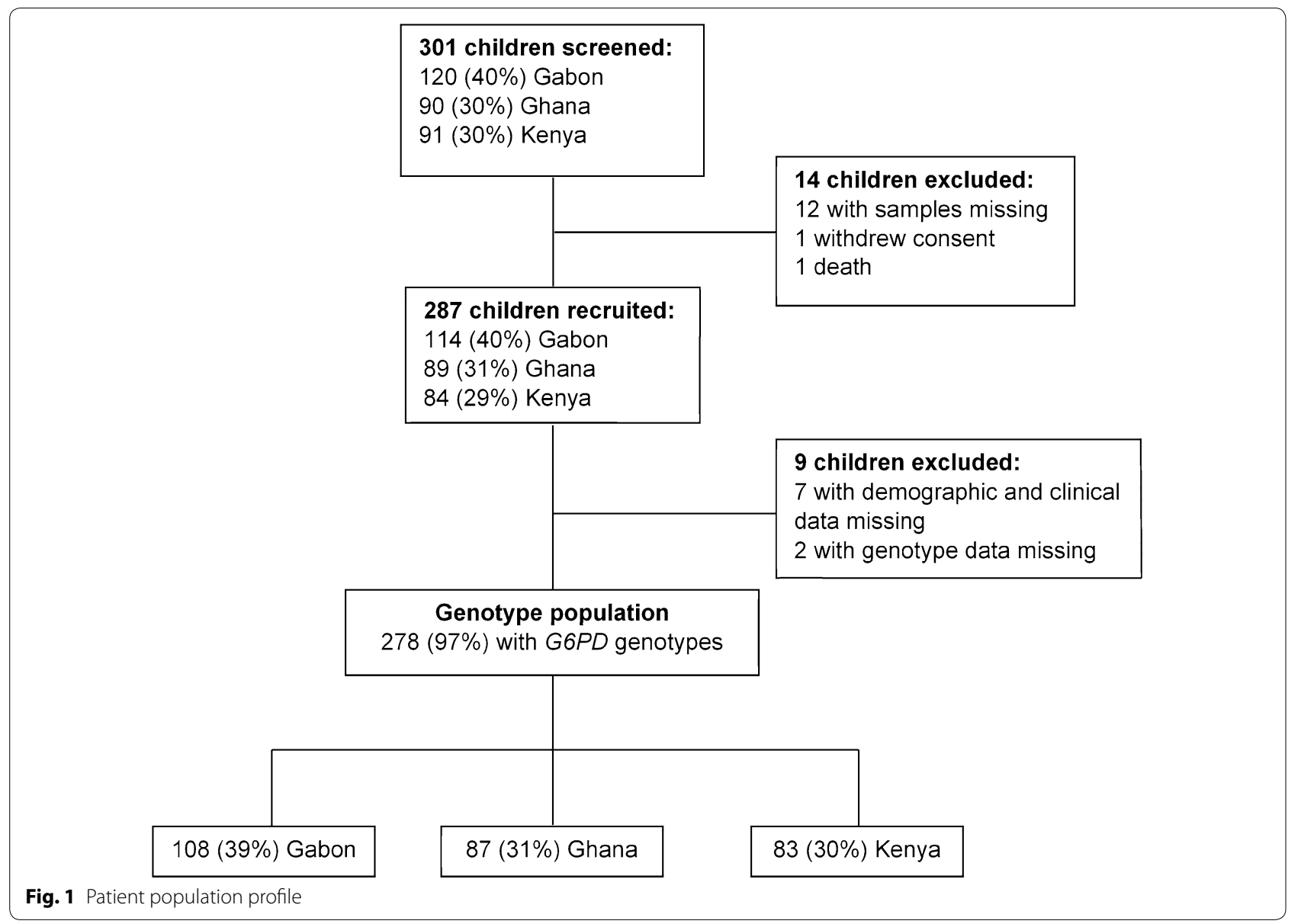

Table 2 Frequency of G6PD genotypes in malaria children from the three study centres

\begin{tabular}{lllllllllllll}
\hline Country & Centre & Male, N & B & A+ & A- & Female, N & BB & BA+ & A+A+ & BA- & A+A- & A-A- \\
\hline Gabon & Lambaréné & 52 & $28(54)$ & $9(17)$ & $15(29)$ & 56 & $20(35)$ & $14(25)$ & $2(4)$ & $1(2)$ & $17(30)$ & $2(4)$ \\
Ghana & Kumasi & 46 & $25(54)$ & $8(18)$ & $13(28)$ & 41 & $10(24)$ & $11(27)$ & $2(5)$ & $1(2)$ & $15(37)$ & $2(5)$ \\
Kenya & Kisumu & 48 & $31(65)$ & $13(27)$ & $4(8)$ & 35 & $22(63)$ & $7(20)$ & $0(0)$ & $0(0)$ & $6(17)$ & $0(0)$ \\
Total & & 146 & $84(58)$ & $30(20)$ & $32(22)$ & 132 & $52(39)$ & $32(24)$ & $4(3)$ & $2(2)$ & $38(29)$ & $4(3)$ \\
\hline
\end{tabular}

Data are shown as $\mathrm{N}(\%)$. G6PD genotype: male normal $=\mathrm{A}+$ or $\mathrm{B}$; male hemizygous $=\mathrm{A}-$; female normal $=\mathrm{BB}$ or $\mathrm{BA}+$ or $\mathrm{A}+\mathrm{A}+$; female heterozygous $=\mathrm{BA}-$ or $\mathrm{A}+\mathrm{A}-$; female homozygous $=\mathrm{A}-\mathrm{A}-$

by study site are shown. Between the study centres, there was a significant difference $(p<0.0001)$ in the prevalence of $\mathrm{A}^{-} \mathrm{G} 6 \mathrm{PD}$ deficiency among males. Among females, the frequency of $\mathrm{A}^{-} \mathrm{G} 6 \mathrm{PD}$ deficiency was lower compared to males. The prevalence was $4 \%$ in Lambaréné (Gabon), $5 \%$ in Kumasi (Ghana) and $0 \%$ in Kisumu (Kenya).

To investigate the association of G6PD genotypes with baseline variables, the children were grouped based on gender and G6PD genotype. Baseline demographic and clinical data are given in Table 3. Using a multivariate regression analysis adjusted for age, gender, centre, weight, temperature and parasitaemia, there was a significant association of the G6PD genotypes with the adjusted mean baseline of haemoglobin concentrations $(p<0.0001)$. Furthermore, a comparison between children G6PD normal and heterozygous $(p<0.0001)$, and between individuals G6PD normal and G6PD deficient $(p=0.009)$ showed a significant difference in adjusted mean baseline haemoglobin. The G6PD mutant 
Table 3 Baseline demographic and clinical data following G6PD genotypes

\begin{tabular}{|c|c|c|c|c|c|c|}
\hline Characteristic & $\begin{array}{l}\text { Male normal } \\
(n=114)\end{array}$ & $\begin{array}{l}\text { Male hemizygous } \\
(\mathrm{n}=32)\end{array}$ & $\begin{array}{l}\text { Female normal } \\
(n=88)\end{array}$ & $\begin{array}{l}\text { Female heterozy- } \\
\text { gous }(n=40)\end{array}$ & $\begin{array}{l}\text { Female homozy- } \\
\text { gous }(n=4)\end{array}$ & $\begin{array}{l}\text { All children } \\
(n=278)\end{array}$ \\
\hline Age, years [range] & $3.3(2.8)[0-10]$ & $2.6(2.2)[0-8]$ & $2.2(1.9)[0-8]$ & $2.3(2.6)[0-10]$ & $2.5(3)[0-6]$ & $2.7(2.5)[0-10]$ \\
\hline $0-3, n(\%)$ & $65(57)$ & $23(72)$ & $69(78)$ & $30(75)$ & $2(50)$ & $189(68)$ \\
\hline $4-7, \mathrm{n}(\%)$ & $39(34)$ & $8(25)$ & $18(21)$ & $7(18)$ & $2(50)$ & $74(27)$ \\
\hline$\geq 8, \mathrm{n}(\%)$ & $10(9)$ & $1(3)$ & $1(1)$ & $3(7)$ & $0(0)$ & $15(5)$ \\
\hline Weight, kg & $15.6(5.2)$ & $12.6(4.7)$ & $13.2(4)$ & $13.2(5.9)$ & $16.3(2.7)$ & $14.1(5)$ \\
\hline $\begin{array}{l}\text { Parasitemia per } \mu \mathrm{L}, \text { geo- } \\
\text { metric mean [range] }\end{array}$ & $\begin{array}{c}150,464[6240- \\
1,270,080]\end{array}$ & $\begin{array}{l}95,369[8153- \\
709,400]\end{array}$ & $\begin{array}{l}138,296[5377- \\
1,677,780]\end{array}$ & $\begin{array}{l}105,520[6380- \\
745,100]\end{array}$ & $\begin{array}{l}172,340[32,176- \\
397,440]\end{array}$ & $\begin{array}{c}132,349[5377- \\
1,677,780]\end{array}$ \\
\hline Temperature, ${ }^{\circ} \mathrm{C}$ & $37.9(1.1)$ & $38.4(1)$ & $38.2(1.1)$ & $38(1.2)$ & $37.7(1.3)$ & $38.1(1.1)$ \\
\hline $\begin{array}{l}\text { Haemoglobin, g/dL } \\
\text { (years) }\end{array}$ & $8.9(2.4)$ & $7.8(2.3)$ & $8.9(2.3)$ & $7.1(2.3)$ & $8.2(1.4)$ & $8.5(2.4)$ \\
\hline $0-3$ & $8.2(2.4)$ & $7.6(2.1)$ & $8.6(2.3)$ & $6.7(2.2)$ & $8.1(2.1)$ & $8.1(2.4)$ \\
\hline $4-7$ & $9.9(2)$ & $8.2(2.8)$ & $9.7(1.8)$ & $6.9(2)$ & $8.3(1.3)$ & $9.3(2.2)$ \\
\hline$\geq 8$ & $9.8(1.8)$ & NA & NA & $10.6(0.6)$ & NA & $10.2(1.7)$ \\
\hline Haematocrit, \% & $27.4(7.3)$ & $23.8(7.1)$ & $27.3(7)$ & $21.7(7.6)$ & $25.5(4)$ & $26.1(7.5)$ \\
\hline $\begin{array}{l}\text { Red blood cell count, } \\
\text { 10^12/L }\end{array}$ & $3.8(1)$ & $3.3(1.2)$ & $3.6(1)$ & $3(1)$ & $3.1(0.4)$ & $3.6(1.1)$ \\
\hline Platelet count, 10^9/L & $151.6(230.4)$ & $98(82)$ & $117.3(118.8)$ & $111(77.7)$ & $63.3(30)$ & $127.5(167.9)$ \\
\hline $\begin{array}{l}\text { White blood cell count, } \\
10^{\wedge} 9 / \mathrm{L}\end{array}$ & $9.4(4.2)$ & $11.6(7.9)$ & $9.8(4.3)$ & $11.6(4.4)$ & $7.7(3.4)$ & $10.1(4.9)$ \\
\hline
\end{tabular}

Values are mean (SD) [range] unless otherwise indicated. G6PD genotype: male normal = A or $B$; male hemizygous $=A-;$ female normal $=B / B$ or $B / A$ or $A / A$; female homozygous $=\mathrm{A}-/ \mathrm{A}-;$ female heterozygous $=\mathrm{B} / \mathrm{A}-$ or $\mathrm{A} / \mathrm{A}-$

$N A$ not applicable; $S D$ standard deviation

genotypes were associated with a $2.7 \mathrm{~g} / \mathrm{dL}$ and $1 \mathrm{~g} / \mathrm{dL}$ decrease in haemoglobin levels in heterozygous and deficient children, respectively. The multivariate regression analysis adjusted for age, gender, centre, weight, haemoglobin levels and temperature did not result in any association $(p=0.29)$ of the G6PD genotypes on adjusted mean baseline parasite densities. In addition, there was no difference in adjusted log mean parasite densities between G6PD normal and heterozygous individuals $(p=0.18)$, and between G6PD normal and deficient children $(p=0.07)$.

\section{Severe malarial anaemia and cerebral malaria}

Following the WHO guidelines [23] which defines severe malarial anaemia (SMA) as $\mathrm{Hb}<5 \mathrm{~g} / \mathrm{dL}, 26$ children (9 \%) were affected by SMA in this study. The mean haemoglobin concentrations was $4 \mathrm{~g} / \mathrm{dL}$ (range $1.9-4.9 \mathrm{~g} / \mathrm{dL}$ ). There was no difference $(p=0.66)$ of the haemoglobin levels between SMA children with different G6PD genotypes. However, SMA occurred more frequently among G6PD normal 15/26 (58 \%) compared to G6PD heterozygous 8/26 (31\%) and G6PD deficient 3/26 (12\%) children.

Only 13 patients $(5 \%)$ had cerebral malaria. Nine of them were G6PD normal and four were female G6PD heterozygous.

\section{Discussion}

Glucose-6-phosphate dehydrogenase deficiency has raised in frequencies in malaria-endemic settings as a consequence of the evolutionary pressure exerted by malaria on the human genome [16]. A plethora of previous studies have indicated and suggested a correlation between malaria endemicity and the occurrence of G6PD deficiency (reviewed in [25]).

The main objective of this study was to assess the distribution of G6PD genotypes among African children from three geographically countries presenting with severe malaria and participating in the SMAC clinical trial on different artesunate treatment regimens. While meanwhile many G6PD variants have been described [8, $11]$, the main focus was on the most relevant three variants (376A (G6PD type B, no deficiency), 376G (moderate G6PD deficiency type $\mathrm{A}^{+}$) and 202A severe G6PD deficiency type $\mathrm{A}^{-}$) in Africa [26].

The prevalence of severe G6PD deficiency as determined genetically was higher compared to previous findings from Gabon with $17 \%$ in the present study versus $14 \%$ reported earlier among males and 7 vs $2 \%$ among females [27]. In Ghana $28 \%$ were observed in this study, compared to $9 \%$ among males and 5 vs $3 \%$ among females indicated previously [28]. However, in Kenya the prevalence of severe G6PD deficiency among 
females was lower compared to previous reports with 0 vs $5 \%$ and equal among males 8 vs $8 \%$ [29]. Regardless of the site, G6PD deficiency was considerably higher among males (22\%) compared to females (3\%), with an overall prevalence of $13 \%$ across sites. This finding confirms that males are affected by this blood disorder rather than females and that G6PD deficient females are rather uncommon [26].

A significant association of moderately and severely deficient G6PD genotypes and haemoglobin levels according to the baseline data was observed. In fact, compared to children with the normal G6PD genotype (haemoglobin median: $9.3 \mathrm{~g} / \mathrm{dL}$ ), G6PD heterozygous (haemoglobin median: $6.6 \mathrm{~g} / \mathrm{dL}$ ) and deficient (haemoglobin median: $8.3 \mathrm{~g} / \mathrm{dL}$ ) children had 2.7 and $1 \mathrm{~g} / \mathrm{dL}$ lower haemoglobin concentrations, respectively. The results contradict previous findings, which did not observe any association between G6PD genotypes and haemoglobin levels [30-32]. While in their study, May et al. [32] found lower levels of haemoglobin in G6DP deficient individuals, the association was not significant. An explanation could be the different designs of that and the present study. Here, patients with severe malaria were included, which implies high parasitaemia and haemoglobin concentrations $<5 \mathrm{~g} / \mathrm{dL}$, whereas in the above cited study, the authors excluded patients with haemoglobin concentrations $\leq 7 \mathrm{~g} / \mathrm{L}$ [30] and recruited individuals only with uncomplicated malaria [31] or who were asymptomatically infected [32].

In the case of SMA, there was no difference of haemoglobin levels among children with different G6PD genotypes. This may suggest that G6PD deficiency is not associated with this complication. However, this is in fact surprising especially as a significant difference of baseline haemoglobin values between G6PD normal and deficient individuals was observed. Moreover, in a large case-control study in Kenyan individuals, a significant increased risk to severe malarial anaemia associated with lower haemoglobin levels in G6PD deficient children with severe malaria at the time of hospital admission was found [33]. The likely explanation could be due to the sample size which was rather small.

Although not significant, parasite densities were lower in G6PD deficient children than in G6PD heterozygous, compared to G6PD normal individuals. However, this trend is in agreement with previous works [34, 35]. Conflicting results have been reported regarding parasitaemia and the various G6PD genotypes. Other studies have indicated a significantly lower parasitaemia associated with female G6PD heterozygous individuals, suggesting a protective advantage by this genotype [36-39].

In comparison to individuals with uncomplicated malaria, this study population was hyperparasitemic according to the criteria of severe malaria provided by WHO with lower parasitaemia in G6PD deficient individuals. However, either in uncomplicated or in severe/complicated malaria, G6PD deficiency was always associated with lower parasitaemia [30, 33]. Although the underlying genetic mechanisms are not completely clear, mechanisms suggested are impaired growth of $P$. falciparum parasites in G6PD deficient red blood cells [40] and slow rates of parasite replication [41, 42], more efficient clearance of infected red blood cells [43], and lower abundance of $P$. falciparum 6-phosphogluconolactonase mRNA in parasites from G6PD deficient individuals [44] both uncomplicated and severe malaria.

Several limitations apply to this study. Although some of the observations are in agreement with previous findings, the study group is rather small. The focus was only on the G6PD mutations $202 \mathrm{G}>\mathrm{A}$ and $376 \mathrm{~A}>\mathrm{G}$, although other variants such as A542T, G680T or T968C have also been reported to contribute to G6PD deficiency, albeit at far lower frequencies. The associations observed between G6PD genotypes and the baseline clinical parameters at admission could in fact also be the result of different circumstances. First, only patients with severe malaria were included, which could explain the findings, especially as the G6PD deficient allele 202A appears to confer protection against cerebral malaria and increases the risk of severe malarial anaemia $[16,17]$. Second, depending on the mechanism of protection, G6PD deficiency might be associated with delayed presentation to the hospital and be a plausible explanation of the differences observed in haemoglobin concentrations at first admission. Third, alpha-thalassaemia, another haemoglobinopathy which has been associated with lower haemoglobin levels in alpha-thalassaemic Nigerian children and adults [45] cannot be ruled out as a confounding variable as it was not investigated in this study. The statistical analysis using multivariable regression model is in part subjective due to the adjusted variables added.

\section{Conclusions}

G6PD deficiency is more common among children from Gabon and Ghana than in Kenya. A significant association of the G6PD genotypes studied to lower haemoglobin levels was observed, suggesting a possible contributions of G6PD deficiency to the reduced production of erythrocytes in affected individuals. This was, however, not related to severe malarial anaemia experienced by some children. There was no evidence of a significant association between lower parasitaemia observed in G6PD deficient individuals compared to normal ones. 


\section{Abbreviations}

G6PD: glucose-6-phosphate dehydrogenase; ARS: artesunate; SMAC: severe malaria in African children; WHO: World health organization; NADPH: nicotinamide adenine dinucleotide phosphate; SMA: severe malarial anaemia.

\section{Authors' contributions}

TPV designed the experiments, supervised the experiments and data analysis. $A A A, T A, B R O$ are involved in patient recruitment from respective study sites and are principal investigators for SMAC study. PGK contributed to study design and materials. CNN performed the experiments and drafted the manuscript. TPV and CGM contributed in writing the manuscript. All authors read and approved the final manuscript.

\section{Author details}

${ }^{1}$ Institute of Tropical Medicine, University of Tübingen, Wilhelmstrasse 27, 72074 Tübingen, Germany. ${ }^{2}$ Vietnamese-German Center for Medical Research, Hanoi, Vietnam. ${ }^{3}$ Centre de Recherches Médicales de Lambaréné, Lambaréné, Gabon. ${ }^{4}$ Department of Physiology, School of Medical Sciences, University of Science and Technology, Kumasi, Ghana. ${ }^{5}$ Departments of Child Health and Medicine, Komfo Anokye Teaching Hospital, Kumasi, Ghana. ${ }^{6}$ Centre for Clinical Research, Kenya Medical Research Institute, Kisumu, Kenya. ${ }^{7}$ Fondation Congolaise pour la Recherche Médicale, Brazzaville, Republic of Congo.

\section{Acknowledgements}

The authors acknowledge Velia Grummes for technical help during sequencing procedures.

\section{Competing interests}

The authors declare that they have no competing interests.

\section{Availability of data and materials}

All relevant data are within the paper.

\section{Ethics approval and consent to participate}

The study was conducted in accordance with Good Clinical Practices, and approved by authorities for each study site (the Regional Ethics Committee in Lambaréné (CERIL) for Gabon, Committee on Human Research, Publication and Ethics, Kwame Nkrumah University of Science and Technology (KNUST), Kumasi, for Ghana and the National Ethics Research Committee, Kenya Medical Research Institute (KEMRI) for Kenya). Children were enrolled into this study if a parent or guardian was willing to provide written informed consent in accordance with local practice.

\section{Funding}

The authors acknowledge the financial support from fortüne Grant (2270-0) from University Klinikum Tübingen and DFG Grant (DFG Ku 775/17-1) for German-African Cooperation project in Infectiology.

Received: 11 March 2016 Accepted: 15 June 2016

Published online: 07 July 2016

\section{References}

1. WHO. World malaria report. Geneva: World Health Organization; 2015.

2. WHO. Global plan for artemisinin resistance containment (GPARC). Geneva: World Health Organization; 2011.

3. Murray CJ, Rosenfeld LC, Lim SS, Andrews KG, Foreman KJ, Haring D, et al. Global malaria mortality between 1980 and 2010: a systematic analysis. Lancet. 2012;379:413-31.

4. White NJ. The role of anti-malarial drugs in eliminating malaria. Malar J. 2008;7(Suppl 1):S8.

5. Wells TN, Burrows JN, Baird JK. Targeting the hypnozoite reservoir of Plasmodium vivax: the hidden obstacle to malaria elimination. Trends Parasitol. 2010;26:145-51.

6. Howes RE, Battle KE, Satyagraha AW, Baird JK, Hay SI. G6PD deficiency: global distribution, genetic variants and primaquine therapy. Adv Parasitol. 2013:81:133-201.
7. Khim N, Benedet C, Kim S, Kheng S, Siv S, Leang R, et al. G6PD deficiency in Plasmodium falciparum and Plasmodium vivax malaria-infected Cambodian patients. Malar J. 2013;12:171.

8. Cappellini MD, Fiorelli G. Glucose-6-phosphate dehydrogenase deficiency. Lancet. 2008;371:64-74.

9. WHO Working Group. Glucose-6-phosphate dehydrogenase deficiency. Bull World Health Organ. 1989;67:601-11.

10. Beutler E. Glucose-6-phosphate dehydrogenase deficiency: a historical perspective. Blood. 2008;111:16-24.

11. Minucci A, Moradkhani K, Hwang MJ, Zuppi C, Giardina B, Capoluongo E. Glucose-6-phosphate dehydrogenase (G6PD) mutations database: review of the "old" and update of the new mutations. Blood Cells Mol Dis. 2012;48:154-65.

12. Ruwende C, Khoo SC, Snow RW, Yates SN, Kwiatkowski D, Gupta S, et al. Natural selection of hemi- and heterozygotes for G6PD deficiency in Africa by resistance to severe malaria. Nature. 1995;376:246-9.

13. Bwayo D, Kaddumukasa M, Ddungu H, Kironde F. Prevalence of glucose6-phosphate dehydrogenase deficiency and its association with Plasmodium falciparum infection among children in Iganga distric in Uganda. BMC Res Notes. 2014:7:372.

14. Clark TG, Fry AE, Auburn S, Campino S, Diakite M, Green A, et al. Allelic heterogeneity of G6PD deficiency in West Africa and severe malaria susceptibility. Eur J Hum Genet. 2009;17:1080-5.

15. De AC, Migot-Nabias F, Guitard J, Pelleau S, Vulliamy T, Ducroca R. The role of the G6PD AEth376G/968C allele in glucose-6-phosphate dehydrogenase deficiency in the seerer population of Senegal. Haematologica. 2006:91:262-3

16. Malaria Genomic Epidemiology Network. Reappraisal of known malaria resistance loci in a large multicenter study. Nat Genet. 2014:46:1197-204.

17. Shah SS, Rockett KA, Jallow M, Sisay-Joof F, Bojang KA, Pinder M, et al. Het erogeneous alleles comprising G6PD deficiency trait in West Africa exert contrasting effects on two major clinical presentations of severe malaria. Malar J. 2016:15:13

18. Kremsner PG, Adegnika AA, Hounkpatin AB, Zinsou JF, Taylor TE, Chimalizeni $Y$, et al. Intramuscular artesunate for severe malaria in African children: a multicenter randomized controlled trial. PLoS Med. 2016;13:e1001938.

19. Planche T, Krishna S, Kombila M, Engel K, Faucher JF, Ngou-Milama E, et al. Comparison of methods for the rapid laboratory assessment of children with malaria. Am J Trop Med Hyg. 2001;65:599-602.

20. Shah SS, Macharia A, Makale J, Uyoga S, Kivinen K, Craik R, et al. Genetic determinants of glucose-6-phosphate dehydrogenase activity in Kenya. BMC Med Genet. 2014;15:93.

21. Kremsner PG, Valim C, Missinou MA, Olola C, Krishna S, Issifou S, et al. Prognostic value of circulating pigmented cells in African children with malaria. J Infect Dis. 2009;199:142-50.

22. Taylor T, Olola C, Valim C, Agbenyega T, Kremsner P, Krishna S, et al. Standardized data collection for multi-center clinical studies of severe malaria in African children: establishing the SMAC network. Trans R Soc Trop Med Hyg. 2006;100:615-22.

23. World Health Organization. Communicable diseases cluster. severe Falciparum malaria. Trans R Soc Trop Med Hyg. 2000;94(Suppl 1):S1-90.

24. WHO. Management of severe malaria-a practical handbook. 3rd ed. Geneva: World Health Organization; 2013.

25. Luzzatto L, Nannelli C, Notaro R. Glucose-6-Phosphate Dehydrogenase Deficiency. Hematol Oncol Clin North Am. 2016;30:373-93.

26. Guindo A, Fairhurst RM, Doumbo OK, Wellems TE, Diallo DA. X-linked G6PD deficiency protects hemizygous males but not heterozygous females against severe malaria. PLoS Med. 2007;4:e66.

27. Mombo LE, Ntoumi F, Bisseye C, Ossari S, Lu CY, Nagel RL, et al. Human genetic polymorphisms and asymptomatic Plasmodium falciparum malaria in Gabonese schoolchildren. Am J Trop Med Hyg. 2003;68:186-90

28. Burchard GD, Browne EN, Sievertsen J, May J, Meyer CG. Spleen size determined by ultrasound in patients with sickle cell trait, $\mathrm{HbAC}$ trait and glucose-6-phosphate-dehydrogenase deficiency in a malaria hyperendemic area (Ashanti Region, Ghana). Acta Trop. 2001:80:103-9.

29. Suchdev PS, Ruth LJ, Earley M, Macharia A, Williams TN. The burden and consequences of inherited blood disorders among young children in western Kenya. Matern Child Nutr. 2014;10:135-44. 
30. Carter N, Pamba A, Duparc S, Waitumbi JN. Frequency of glucose-6-phosphate dehydrogenase deficiency in malaria patients from six African countries enrolled in two randomized anti-malarial clinical trials. Malar J. 2011;10:241.

31. Enevold A, Lusingu JP, Mmbando B, Alifrangis M, Lemnge MM, Bygbjerg IC, et al. Reduced risk of uncomplicated malaria episodes in children with alpha+-thalassemia in northeastern Tanzania. Am J Trop Med Hyg. 2008;78:714-20.

32. May J, Meyer CG, Grossterlinden L, Ademowo OG, Mockenhaupt FP, Olumese PE, et al. Red cell glucose-6-phosphate dehydrogenase status and pyruvate kinase activity in a Nigerian population. Trop Med Int Health. 2000:5:119-23.

33. Uyoga S, Ndila CM, Macharia AW, Nyutu G, Shah S, Peshu N, et al. Glucose-6-phosphate dehydrogenase deficiency and the risk of malaria and other diseases in children in Kenya: a case-control and a cohort study. Lancet Haematol. 2015;2:e437-44.

34. Martin SK, Miller LH, Alling D, Okoye VC, Esan GJ, Osunkoya BO, et al. Severe malaria and glucose-6-phosphate-dehydrogenase deficiency: a reappraisal of the malaria/G-6-PD hypothesis. Lancet. 1979;1:524-6.

35. Nkuo-Akenji TK, Wepngong P, Akoachere JF. Effects of ABO/Rh blood groups, G-6-PD enzyme activity and haemoglobin genotypes on malaria parasitaemia and parasite density. Afr J Health Sci. 2004;11:93-7.

36. Luzzatto L, Bienzle U. The malaria/G-6-PD hypothesis. Lancet. 1979;1:1183-4.

37. Bienzle U, Ayeni O, Lucas AO, Luzzatto L. Glucose-6-phosphate dehydrogenase and malaria. Greater resistance of females heterozygous for enzyme deficiency and of males with non-deficient variant. Lancet. 1972;1:107-10
38. Gilles HM, Fletcher KA, Hendrickse RG, Lindner R, Reddy S, Allan N. Glucose-6-phosphate-dehydrogenase deficiency, sickling, and malaria in African children in South Western Nigeria. Lancet. 1967;1:138-40.

39. Allison AC, Clyde DF. Malaria in African children with deficient erythrocyte glucose-6-phosphate dehydrogenase. BMJ. 1961;1:1346-9.

40. Luzzatto L, Usanga FA, Reddy S. Glucose-6-phosphate dehydrogenase deficient red cells: resistance to infection by malarial parasites. Science. 1969;164:839-42.

41. Manjurano A, Sepulveda N, Nadjm B, Mtove G, Wangai H, Maxwell C, et al. African glucose-6-phosphate dehydrogenase alleles associated with protection from severe malaria in heterozygous females in Tanzania. PLoS Genet. 2015;11:e1004960.

42. Miller J, Golenser J, Spira DT, Kosower NS. Plasmodium falciparum: thiol status and growth in normal and glucose-6-phosphate dehydrogenase deficient human erythrocytes. Exp Parasitol. 1984;57:239-47.

43. Cappadoro M, Giribaldi G, O'Brien E, Turrini F, Mannu F, Ulliers D, et al. Early phagocytosis of glucose-6-phosphate dehydrogenase (G6PD)deficient erythrocytes parasitized by Plasmodium falciparum may explain malaria protection in G6PD deficiency. Blood. 1998;92:2527-34.

44. Sodeinde O, Clarke JL, Vulliamy TJ, Luzzatto L, Mason PJ. Expression of Plasmodium falciparum G6PD-6PGL in laboratory parasites and in patient isolates in G6PD-deficient and normal Nigerian children. Br J Haematol. 2003;122:662-8.

45. Mockenhaupt FP, Falusi AG, May J, Ademowo OG, Olumese PE, Meyer CG, et al. The contribution of alpha+-thalassaemia to anaemia in a Nigerian population exposed to intense malaria transmission. Trop Med Int Health. 1999:4:302-7.

\section{Submit your next manuscript to BioMed Central and we will help you at every step:}

- We accept pre-submission inquiries

- Our selector tool helps you to find the most relevant journal

- We provide round the clock customer support

- Convenient online submission

- Thorough peer review

- Inclusion in PubMed and all major indexing services

- Maximum visibility for your research

Submit your manuscript at www.biomedcentral.com/submit
() Biomed Central 